\title{
Design and Implementation of a Web-Based Virtual Classroom System
}

\author{
Akinyokun Oluwole Charles, Iwasokun Gabriel Babatunde \\ Department of Computer Science Federal University of Technology, Akure, Nigeria
}

\begin{abstract}
Despite the increasing growth in popularity of virtual classrooms and online-learning, some factors which include limited bandwidth for audio and video, poor transmission quality, poor collaborative work and limited feedback during virtual classroom sessions still need to be addressed. This paper presents the design and implementation of a virtual classroom system that focuses on collaborative learning between students and tutors at remote locations. The different components of the system allows students to engage in group activities and collaborate with instructors in a commonly shared windows on which text, audio or video objects are added and shared online. The system was developed and hosted on the web using Moodle, Elluminate, WAMP Server, Java Script, MySQL, PHP and Dreamweaver. A practical demonstration was carried out using an Undergraduate Course of the Department of Computer Science in the School of Sciences, Federal University of Technology, Akure, Nigeria. The results obtained from the demonstration exhibit the suitability and adequacy of the virtual classroom system. The evaluation of the system based on selected indices also shows its level of performance and ratings.
\end{abstract}

Keywords: Chat Module, Elluminate Module, Enterprise Information System, Instructor and Participant, Virtual Classroom,

\section{Introduction}

Education is the process of bringing about a relatively permanent change in human behaviour. It is the main instrument used to preserve, maintain and upgrade people's culture, norms and values. In any society, education is a veritable tool for determining technological advancement and passing on to the new generations, the existing knowledge of the physical environment. Education also serves as platform for introducing individuals to any organization, giving skills for performing daily jobs, leisure as well as inculcating sound morals in the people for their own benefit and that of the society. In summary, education is the platform on which younger generation understand the past heritage as well as participate and contribute meaningfully to the growth and development of the society.

Conventionally, education has been practiced across the world in segregated buildings by carefully regimented and standardized classes of students and teachers. This has a consequential effect on both the teacher and the learner. For example, education is implicitly restricted only to those who could be in the classroom and follow the teacher rigidly with the pace by which he presents his lectures. Population explosion and mass educational system in every region of the world brought greater challenges to this method of education. For instance, there is problem of inadequate number of human and material resources to cater for the education of the large population. The population of school age citizen in most places has grown tremendously to the extent that only a small percentage can be offered admission. The student - lecturer and student-classroom ratios have grown to the extent that teaching and learning in the classroom have been less effective [1-4]. The field of education therefore provides the most fascinating application of computing system, which has consequently attracted considerable attention from educationists and policy makers since the late 1960s when computers were introduced into classrooms. Various information technologies have been applied in learning and teaching, such as Computer Aided Instruction, (CAI), Computer Aided Learning (CAL), Research Packages, Project Monitoring, Computerized Libraries and so on. As a result of technological advancement in multimedia technology, computer networks and the Internet, computers are now being applied in distance learning premised on on-line and real time teaching and instruction [1].

A distance learning system brings education closer to the people and removes the stress and constraints, which characterize the traditional classroom learning system. The hallmarks of distance learning are the separation of teacher and learner in space and/or time [5], the control of learning by the student rather than the distant instructor [6], and non-contiguous communication between student and teacher, mediated by print or some form of technology [7-8]. At its most basic level, distance education is a process in which significant number of teachings is conducted by remotely located lecturers [1]. Two categories of distance learning systems exist; namely On-line and Off-line distance learning [9]. On-line distance learning, also known as virtual classroom is a type of learning system in which students work on their own at home or at the office and communicate with faculty and other students via e-mail, electronic forums, videoconferencing and other forms 
of computer-based communication. Off-line distance learning on its own is a multi-campus system where a University or Polytechnic establishes many campuses and each of these campuses may be centrally controlled or decentralized. There is little or no difference between the off-line distance learning and traditional learning in that the off-line distance learning system is still characterized by the features of the traditional system. The earliest form of virtual classroom learning took place through correspondence courses in Europe [10]. Many institutions, both public and private, offer university courses for self-motivated individuals through independent study programs. Students work on their own, with supplied course materials, print-based media and postal communication, some form of teleconferencing and/or electronic networking, and learner support from tutors and mentors via telephone or E-mail. Virtual classroom platforms provide collaborative learning facility, which is an integral component of teaching and learning system as well as instant feedback with facilities like e-mail, wikis and bulletin board at the disposal of teacher and the students. Virtual classroom also provides two-way communication with faculties to incorporate many interactive features, for both on-line and off-line use thereby alleviating the lack of sense of belonging of remote students.

\section{Related Works}

The limitations of several virtual classroom systems include lack of interactive response, live streaming and assignment management. An intranet-based virtual classroom system that enhances the interactions between students and teachers is introduced in [11]. The system introduces a new design for e-learning and removes some of the problems of the existing systems as well as increase the interaction between teacher and students with the impression of a real life classroom. The system provides live streaming of lectures while assignments, questions and answers can be uploaded. The authors in [12] proposed a virtual classroom system which incorporates several modules for live video lecture, whiteboard technology, chat-room and several teacherstudents feedback modules. The system is an innovative combination of synchronous learning via live streaming video, audio and chat over the Internet. It is blended with asynchronous online interaction and group works in a way that mimics real life scenarios. Failure to incorporate notifications email and mobile phone through short message service (SMS) are the major limitations of the system.

A virtual classroom system using virtual image creation technique is proposed in [13]. The system comprises of three classrooms. The first classroom is for theory that contains video clips instructions for lecture and practice and online live lectures while the second classroom is a library that connects the e-learning websites concerning radiographic testing. The third and final classroom is a laboratory for observing experiment results. All the three classrooms were designed to be virtual using computer graphic technique. Characters representing learners were also created in order to create interaction between learners and virtual classrooms. The authors in [14] presented an architectural design for an integrated system for the delivery of lectures in a virtual environment. The architecture and description of the system components are presented with the techniques and recommendations for the implementation of the designed system. The architecture is multi-tier, modular, scalable and built for adaptability to database middleware suite. All functionalities within the application are delivered with web services, communicate via industry standard Extensible Mark-up Language (XML) messaging and access is strictly via a web browser. The virtual classroom application utilizes an operating system with facilities for providing users web services, data and pre-processor hypertext with a simple and consistent user interface corresponding to the tiers. With the object-oriented design method, three logical categories were identified for application objects; namely, objects that deal with presentation aspects of the application, objects that deal with the business rules and data, and objects that accept and interpret user requests and control the business objects to fulfill the requests.

The authors in [15] presented a virtual classroom by using Real-Time Messaging Protocol (RTMP) which is a sophisticated TCP-based real-time networking protocol that supports the efficient exchange of messages, synchronized data, audio, and video. The system enables teachers and students to publish streaming audio, video and other data messages to present streaming media with interaction or navigation. On the Internet, the system allows students immediate interaction with teachers with a real classroom impression. A web-based collaborative system that serves as an add-on feature to the learning system for a post class sharing of resources by the teacher and student is also proposed. The web-based virtual classroom system proposed in [16] is based on e-pedagogy with enhanced features for collaboration among participants (instructors and students) in a webbased learning environment. Instructor, course, student and learning performance formed the major objects used in developing the system model, the features of which include course outline, presentation, resources, assessment/opinion poll, chat, hands-up, students' list, course information and collaborate, all harnessed to achieve experiential learning among students.

The authors in [17] presented the design and implementation of a learning technology project developed to advance the wireless technologies for building an ad-hoc classroom in order to contrive a modern and new learning environment. A wireless platform was developed for teacher and students to establish a classroom dynamically irrespective of location and time bounds. As supported in traditional classroom, 
information technology that provides teacher with teaching aids was developed. The aids include blackboard, board rubber, colored chalk, microphone, voice and video recorders, and so on for course teaching and discussions in an ad-hoc classroom. Student was also provided with electronic school bag termed eSchoolbag with electronic packing of book, notebook, parents' contact book, pencil case, writing materials, sheets, calculator, address book and so on. In [18], the primary steps towards building and implementing a successful concept of a virtual classroom are presented. Information on the assessment, planning, design, implementation, and maintenance of a virtual classroom in ten easy steps was explicitly divulged with a summary that failure to follow these steps can ultimately lead to poor, incomplete, inadequate and ineffective solutions to Internet based distance education.

In [19], the authors designed a web-based virtual online classroom system based on learning theories and streaming media technologies. The major components of the system are instructional communicating environment (ICE) and collaborative learning environment (CLE). ICE provides learners with learning materials, lecture videos, interactive environment among others. CLE supports active learning by providing the environment with learning tools and materials and contextual discussion for learners. The environments were designed with event-based synchronous strategies and e-learning technologies standards.

\section{Proposed Virtual Classroom System}

The architecture of the proposed system is presented in Figure 1 with four interrelated modules, namely, Application tiers, Application objects, Data processing and Course.

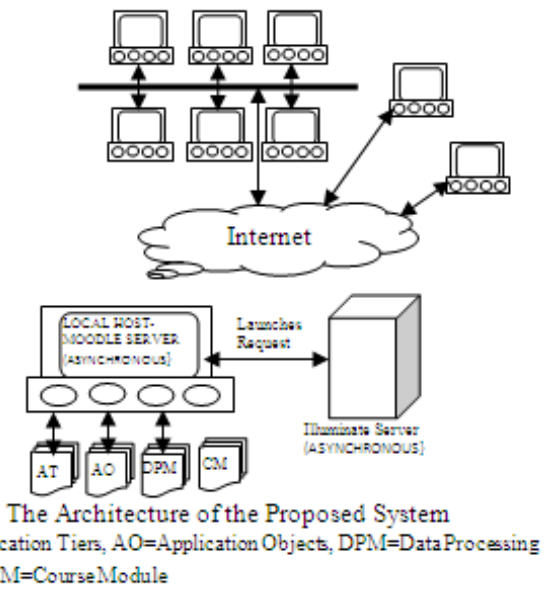

\section{Application tiers}

The application tiers consist of Client-Tier, (CT), Web-Tier (WT) and Enterprise Information SystemTier (EIST) as shown in Figure 2. The CT application is put in place to provide a Graphic User Interface (GUI) to the end users via a web browser, such as Internet Explorer, Opera or Firefox. HTML and PHP with Java applet is used to build the client-side GUI page. A web server, Apache or Internet Information Server (IIS) which is bundled with Microsoft Office server reference implementation, is used to serve the GUI page at the web-tier, and to provide dynamic content HTML/PHP pages. The web-tier holds data model of the business data and presents it to the client through HTML/PHP pages, accepts and analyzes the user's inputs, passes the user's request to the Enterprise tier for processing, and forward response back to the client. The WT handles the business logic and data as well as communicates with the Enterprise Information System (EIS) tier through appropriate protocols. MySQL version 5.2 (a relational database) and PHP were used to build the EIS tier application.

The web-tier application communicates with the Enterprise-Tier (ET) application through a HTML/PHP Interface and a transparent Internet Protocol (IP). This application enables a great flexibility, modularity and independence of the client from the server application development. 

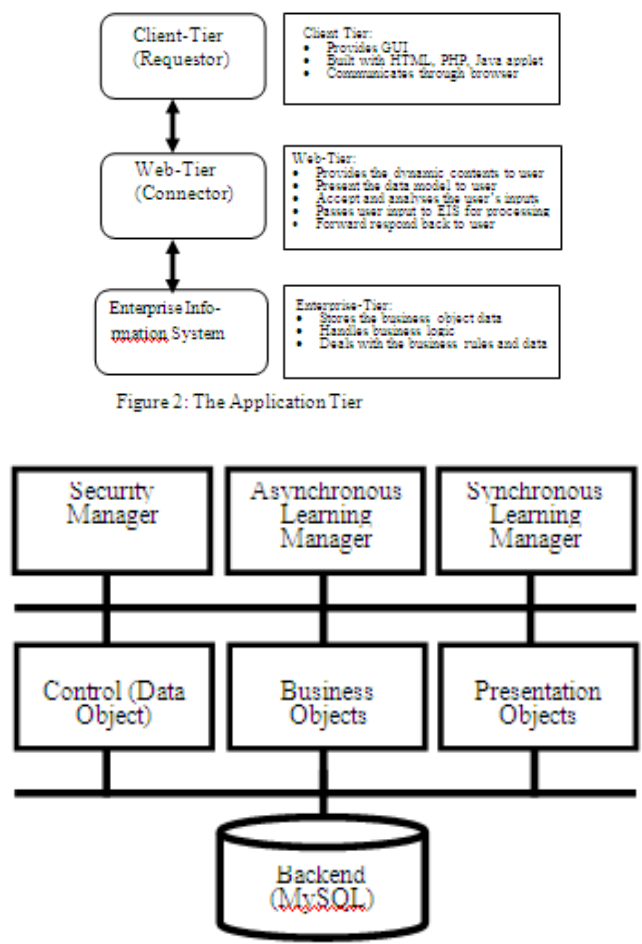

Figure 3: Application objects

\section{Data Processing Architecture}

The proposed system is designed for multi-tier applications and offers a lot of flexibility on the selection of the method for distributing application functionality across the tiers. There is a client tier, which is the browser that communicates with the web tier. There is also the Enterprise Information System (EIS) or the database tier, which holds persistent application data. A choice is made on whether the web tier accesses the EIS resources directly or goes through an Enterprise Java Bean (EJB) tier. This depends on the functionality, complexity, and scalability requirements of the application in the real world. Since such requirements can change as the application evolves, one design goal is to make it amenable to migration to an EJB-centric approach which makes the application a multi-tier. The architecture of a general multi-tier EJB-centric Java 2 Enterprise Edition (J2EE) application illustrating the flow of data through the application is shown in Figure 4.

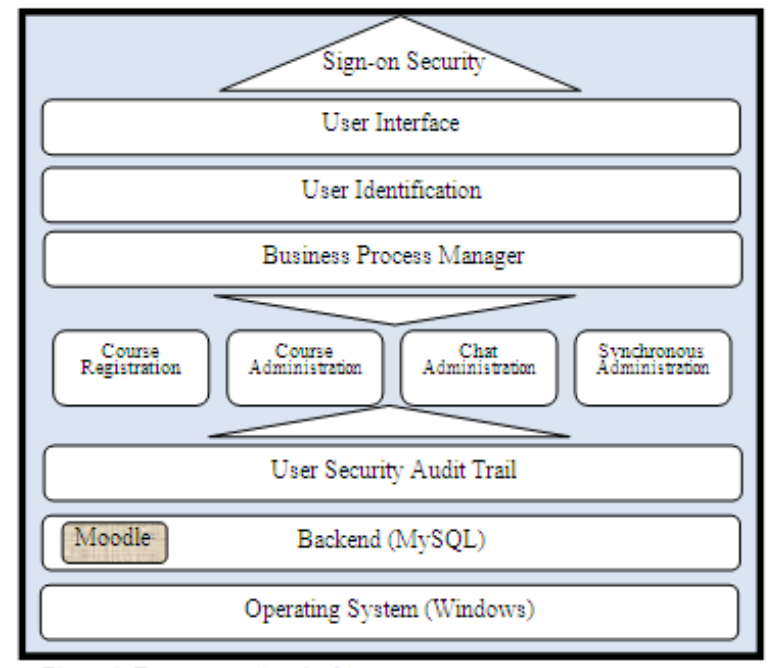

Figure 4: Data processing Architecture

\section{Logical Design}

This application models a web-based student course delivery system and its application interface is presented to the students through a web site where students interact with the application using a web browser. The application presents the student a catalog of courses for selection and registration. New students are 
required to register using administrator assigned username and password. The system notifies the student of a successful registration by a confirmation email using JavaMail API. There is also room for querying what courses have been registered for while lecture is either in asynchronous or synchronous mode.

\section{Usability Specification}

The usability specification is the major ways of using the application. The following are the available functionalities from the user interface of the application:

a. A set of links or navigation bars on each page that provide students with quick access to common navigational tasks.

b. An organized view of the site's contents through a categorized course catalog, which provides students a way to locate courses based on key-word descriptions.

c. A master view of the catalog that lists course of interest to the student resulting in the user navigating through a catalog category or the outcome of a keyword searches.

d. A detailed view of the courses

e. A chatting facilities

f. Access to a forum where users can exchange ideas on different issues relating to a given course

g. Access to submission and uploading of assignment and subsequent access to the graded result

The student module creates and manages the students' course registration information on identification, name, password, address, phone number and email address. The course catalog module returns a list of courses available. The module also returns detail information about the selected course. The registering client module creates a registering cart for the student for the duration of the user's session. The registration-processing module manages interactions while the application determines whether the user is logged in and if not, it either calls the student module to set up a new student or initiate login. The application allows user revision or cancellation of the course selections for registration. Below are the other modules and their responsibilities:

a. Assignment module performs assignment registration processing.

b. Chat module used for live-time discussions in a very efficient way.

c. Glossary module allows a flexible way for students to add or present definitions (and more) that can be linked through the entire class site.

d. Elluminate module [20] used for synchronous lecture delivery via White board, Video, Audio, Application Sharing, Breakout Rooms, Power point Import, Polling, Participant Sort, Emoticons, Close a Session and Hand Raise as tools.

e. Lessons module for the addition of lessons that guide the student based on provided answers.

f. Wiki module similar to a blog (web log or journal), except that everyone can contributes, edit or makes comment and posting on it is not approved by the administrator to allow quick operation.

g. Workshop module creates a workshop space for the class and facilitates a wide range of peer review options.

The interrelationship among the different modules is presented is Figure 5 showing the modular decomposition of the application for its high-level modules shown in Figure 3.

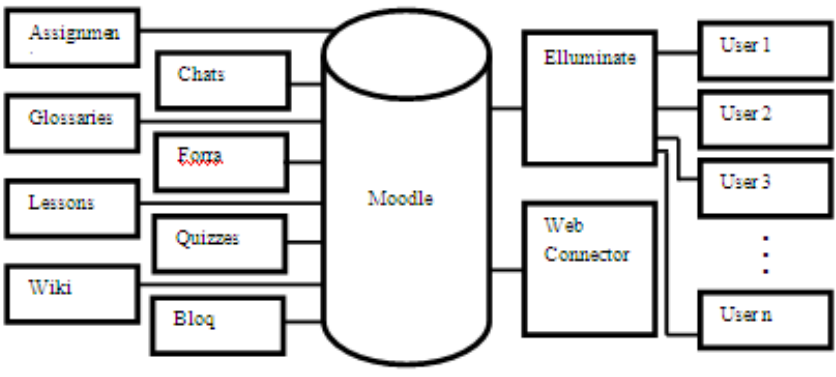

Figure 5: The interrelationship among application modules

\section{Implementation Technique}

The proposed virtual classroom system is modular, scalable and built using the power of MySQL's suite middleware. All functionalities within the application were delivered using Apache and Java J2EE Web Services and communicate via industry standard XML messaging. Access is purely via a web browser and the application utilizes the Windows Apache MySQL and PHP (WAMP) facilities while providing the users with a 
simple and consistent User Interface. The MySQL powerful sign-on technology handles application security. The MySQL's Process Manager records and provides a viewable audit of all message transactions between web services within the system. The frontend is delivered as pure HTML to ensure that it is extremely lightweight and browser independent. The operating system on which the application server is installed is Microsoft Windows Vista with WAMP (Windows, Apache, MySQL and PHP) server 2.0 installations. The installation of WAMP server automatically installed mysq1_essential_5.0.5la_win32.msi, apache 2.2.8 and php_5.2.5 and created WWW folder for providing Internet root directory. The installation of the WAMP server is proceeded by the setting up of Moodle suit [21] by zipping the downloaded Moodle zipped file into a Moodle folder and running the Moodle installer script (install.php) to create the config.php file which is used by the Administrator to setup the site configuration and creation of the home page. To maintain simple and minimum size development, the following assumptions were made during implementation:

a. Application design only focuses on the students 'course registration interaction while administration interaction and the EIS tier database tables were done manually.

b. For simplicity, only the courses offered in the following semester are stored in the database with no limits to the number of students for enrolment. Theoretically, one or more instructors can deliver a course within the same semester, thus, no limit on students.

c. There is no restriction on how many courses a student can enroll.

d. Instructors are not assigned to specific departments

e. Cancellation of a registered course is only performed via the administrator's interface.

The user interface consists of a set of screens delivered to the browser when the user requests an application URL. The following interrelated screens are developed:

a. Entry Login Screen for controlling access to the application and it is launched and accessed through a browser

b. Course Category Screen for selecting the appropriate course for enrolment after successful logon

c. Registered Course Screen for accessing all the chats, assignments, forums and other modules set up by the course administrator or teacher

d. Virtual Classroom Screen for entering the virtual classroom in an online meeting with the lecturer in a prescheduled lecture

After a successful setup of the PHP scripts, the home page for the Virtual Classroom system was created for the Administrator to logon and carry out several tasks including the following:

a. Creation and editing of user's account

b. Course creation and deletion

c. Administration of Instructor/Teacher accounts

d. Editing of the site-wide settings like themes and so on

The virtual classroom was set up for the Federal University of Technology, Akure, Nigeria (FUTA) which has six schools including School of Agriculture and Agricultural Technology (SAAT), School of Engineering and Engineering Technology (SEET) and School of Sciences (SOS). Others are School of Environmental Technology (SET), School of Management Technology (SMAT) and School of Earth and Mineral Sciences (SEMS). Each school has Departments with various programmes on Undergraduate (B. Tech.), Postgraduate Diploma (PGD), Master of Technology (M. Tech.) and Doctor of Philosophy (PhD) degrees. The Department of Computer Science in the School of Sciences was selected for the case study with emphasis on a B. Tech. course; namely Database Systems I (CSC501). However, this does not preclude courses from other Schools, Departments and Programmes from being uploaded. The Administrator accesses the Course Management Module from the Site Administration Block shown in Figure 6. The Schools were setup at the top level of the 'Course' tab while Departments were setup as sub-category within the Colleges. The programmes were setup as sub-category within the Departments and the courses were placed under the programmes as shown in Figure 7. Courses were added, deleted or modified with the 'Add/Edit' button shown in Figure 6. 


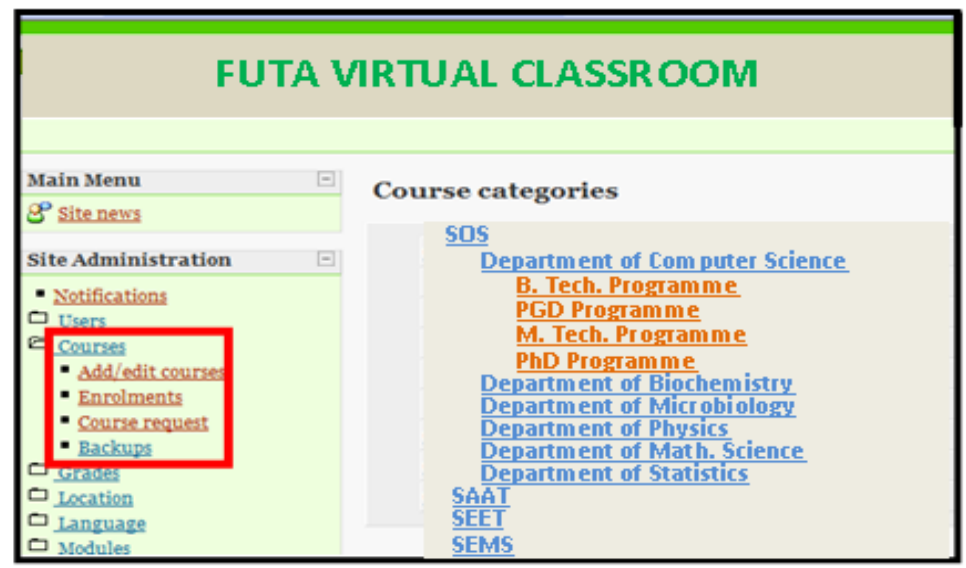

Figure 6: Course Management Module

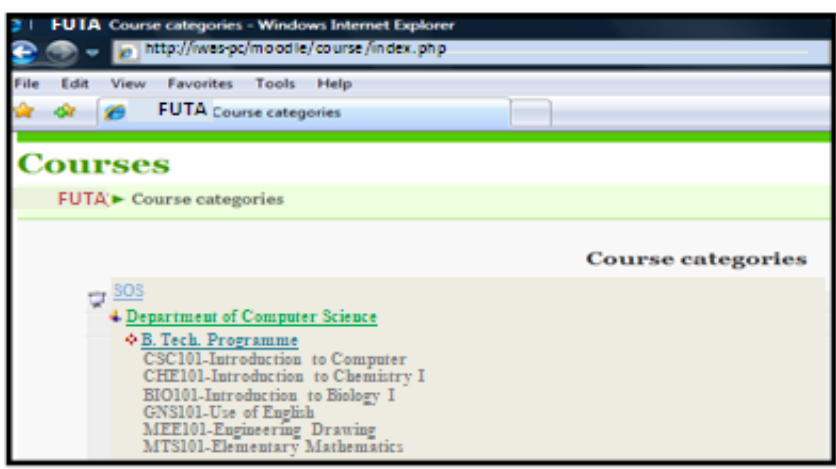

Figure 7: Programme setup window

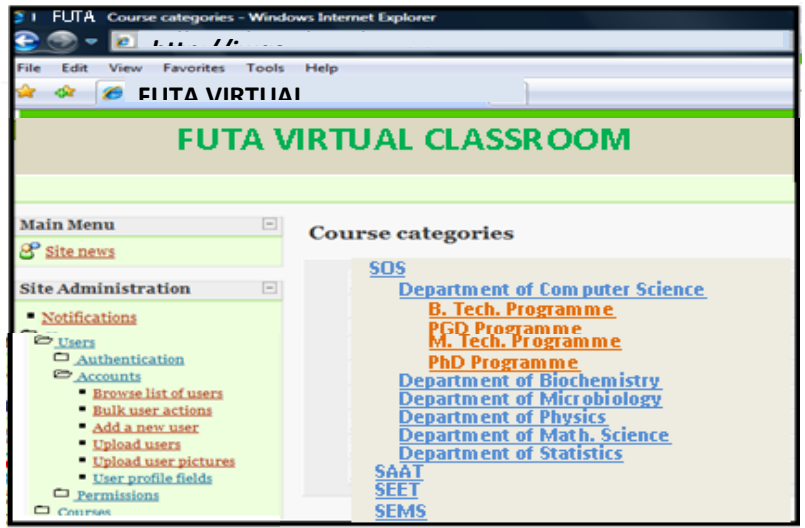

Figure 8: Window for

Clicking on the 'User' button shown in Figure 6 leads to the display of the Course Administration window shown in Figure 8. The 'Add a new user' button is used to add a new user while the "Upload users" button is used to upload user whose record exists in a designated file. The record office of each Department stores records of both old and new students in excels files which were converted to Comma Separated Values (CSV) format to serve as the basic format for uploading files into the virtual classroom system.

The material content of each course including lecture note, quiz, forum, assignment, chat and so on were setup formally on the appropriate menus. The lecture notes were prepared in MS Word and broken into fourteen weeks delivery modules. Assignment and quiz questions were also prepared for each week. A user can be associated with the correct courses using Internal Enrollment (IE), External Databases (ED), Flat File (FF) or Paypal module. The Internal Enrollment module requires that student manually finds and enrolls for his or her courses. 

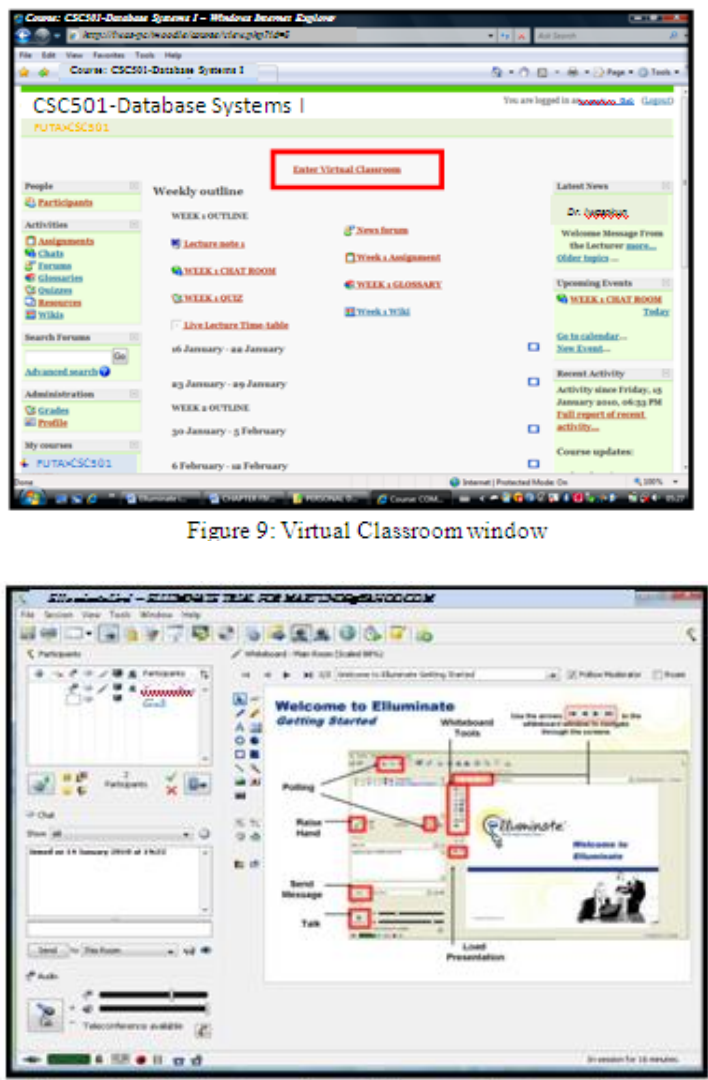

Figure 10: Moderator and participant activation window

As a security measure, each student is assigned an enrollment key for every enrolment. The key is coded to bar un-officially recognized student from gaining access to the Moodle site. The enrollment period is the number of days beginning from the commencement of the course work to its end. After this period, all enrolled students are removed from the file. The system uses Elluminate synchronous platform that was incorporated into Moodle for lecture delivery. CSC501 (Database Systems I) was delivered in synchronous mode as follows:

a. A duly registered student would have access into the virtual classroom after supplying necessary logon parameters. With the asynchronous platform, he/she would be able to see the virtual classroom link that brings out the Time Table for CSC501.

b. Elluminate platform recognizes two categories of users; namely Moderators and Participants. The course lecturer is the moderator while the students are the participants. The virtual classroom for CSC501 has capacity for 50 participants who gained access through the platform's web site via a link labeled "Enter Virtual Classroom" shown in Figure 9.

c. The system has two URLs for conducting the virtual classroom in Elluminate synchronous platform. One is for the participants while the other is for the instructor. The URL for the participant is incorporated into Moodle platform source code, which runs on Elluminate server.

d. Both the instructor and the participants through the URL on the web browser, are equally privileged to activate the virtual classroom. The activation for a participant is shown in Figure 10 and the virtual classroom delivery of lecture for on the uploaded CSC501 power point presentation on the whiteboard is shown in Figure 11.

To verify the efficiency of the system, a formative evaluation of the system was conducted in line with the evaluation indices formulated in [19]. The view and responses of one hundred learners and ten instructors from FUTA were investigated with the aid of a questionnaire that features accessibility, provision of appropriate level of interaction, efficiency of support for learning methods, convenience in use, usefulness for active learning and aid to teaching as the indices for the evaluation of the system's interactive modules. The indices were rated using a scale of 5, 4, 3, 2 and 1 for 'Excellent', 'Very Good', 'Good', Average' and 'Poor' respectively. 


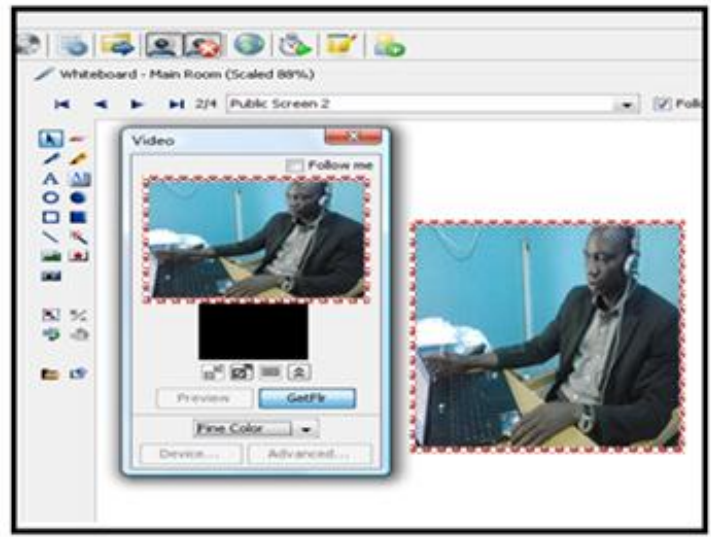

Figure 11:Virtual Classroom delivery of lecture

As shown in Table 1, the learners believe that the 'Chat' module is mostly accessible with average rating of 4.67 while the 'Elluminate' module is the least with average rating of 1.19. On the appropriateness for learning of the different modules, the learners are of the opinion that the 'Chat' module is the best while the 'Elluminate' module is the worst. Table 1 also reveals that the 'Elluminate' module also attracted the least ratings for usefulness for active learning and aid to teaching.

Table 1: Learners Evaluation Results

\begin{tabular}{|l|l|l|l|l|l|l|}
\hline & Assignment & Chat & Glossary & Elluminate & Wiki & Workshop \\
\hline Accessibility & 4.45 & 4.67 & 4.03 & 1.19 & 2.98 & 2.82 \\
\hline $\begin{array}{l}\text { Provision of Appropriate } \\
\text { level of Interaction }\end{array}$ & 4.26 & 4.76 & 3.27 & 2.27 & 2.31 & 2.68 \\
\hline $\begin{array}{l}\text { Efficiency of support for } \\
\text { learning method }\end{array}$ & 4.37 & 4.29 & 3.65 & 2.71 & 4.17 & 3.77 \\
\hline Convenience in use & 3.89 & 3.18 & 2.98 & 3.01 & 4.61 & 3.91 \\
\hline $\begin{array}{l}\text { Usefulness for active } \\
\text { learning }\end{array}$ & 3.67 & 4.74 & 3.12 & 2.59 & 4.17 & 4.54 \\
\hline Aid to teaching & 4.15 & 4.87 & 4.39 & 3.17 & 4.29 & 4.31 \\
\hline
\end{tabular}

Table 2: Instructors Evaluation Results

\begin{tabular}{|l|l|l|l|l|l|l|}
\hline & Assignment & Chat & Lesson & Elluminate & Wiki & Workshop \\
\hline Accessibility & 4.55 & 4.58 & 3.13 & 1.11 & 2.51 & 3.72 \\
\hline $\begin{array}{l}\text { Provision of Appropriate } \\
\text { level of Interaction }\end{array}$ & 3.25 & 3.83 & 1.94 & 1.59 & 3.73 & 3.59 \\
$\begin{array}{l}\text { Efficiency of support for } \\
\text { learning method }\end{array}$ & 4.29 & 4.02 & 3.15 & 3.39 & 2.74 & 3.22 \\
\hline Convenience in use & 4.18 & 4.78 & 4.08 & 2.99 & 4.28 & 2.95 \\
\hline $\begin{array}{l}\text { Usefulness for active } \\
\text { learning }\end{array}$ & 3.82 & 4.65 & 4.14 & 3.65 & 2.41 & 2.45 \\
\hline Aid to teaching & 2.75 & 3.25 & 2.91 & 2.71 & 1.18 & 2.17 \\
\hline
\end{tabular}

Table 2 presents the instructors ratings of the different modules. The investigated instructors are also of the opinion that the 'Chat' modules is the best rated in term of accessibility, provision of appropriate level of interaction, convenience of use, usefulness for active learning and aid to teaching with average rating of 4.58, $3.83,4.78,4.65$ and 3.25 respectively. While the 'Assignment' module also enjoys the best rating in efficiency of support for learning method, the 'Elluminate' module is the least rated in accessibility, provision of appropriate level of interaction and convenience in use with average ratings of $1.11,1.59$ and 2.99 respectively. The 'Wiki' module is the least rated in efficiency of support for learning method and aid to teaching. The highest ratings on most indices enjoyed by the 'Chat' module from both the learners and the instructors imply that it outperformed the other modules in meeting their expectations and satisfactions. The least ratings of the 'Elluminate' module also imply the low performance of the module in meeting up with their expectations and satisfactions. One factor that promoted the highest rating of the 'Chat' module is its instantaneous and interactive nature. The lowest ratings recorded for the 'Elluminate' module is attributed to inadequacies in supports from some external factors such as Internet connectivity and bandwidth for its highest level of sophistication and complexities required for real life video, audio and text display. Real life interactions via the module sometimes failed due to poor Internet connection arising from low VSAT signals. Other causes of failure include congestion and deadlock arising from several online and real time transactions emanating from different sources. 


\section{Conclusion}

The design of a virtual classroom system that serves as a learning platform with events based synchronous and asynchronous modes has been presented. The system consists of several modules which help it to meet up with its designed objectives. The assignments module enables teachers to grade and provide comments for uploaded files and assignments created on and off line, the chats module allows participants to have a real-time synchronous discussion via the web while the glossaries module enables participants to create and maintain a list of definitions in a way similar to the dictionary. The modules for lessons, quizzes and wiki are made up of contents that make them to function optimally in interesting and flexible ways. A whiteboard is also presented as a main presentation window for writing and displaying of images. The audio features allow participation in conversations during a classroom session using microphones and speakers (or headset) via Voice over Internet Protocol (VoIP) while the video features allow the transmission and receipt of video broadcasts in real life and interactive manners.

The different perspective of a group of users has been investigated based on some indices with the results showing significant rating of the system. The investigation revealed that the 'Chat' module outperformed other modules in meeting the users' expectations and satisfactions while the 'Elluminate' modules, which is the core module still need to be greatly improved to increase its present level of performance. This will be the focus of the feature research.

\section{References}

[1]. Akinyokun O.C., (2003), Prototype of Multimedia System for Nigerian University Collaborative Teaching and Learning. Proceedings of All African University Day Seminar, Federal University of Technology, Akure.

[2]. Akintola K. G, (2004), Development of Computer Network for Teaching and Learning in Nigerian Universities.M. Tech. Thesis, Federal University of Technology, Akure.

[3]. Akintola K. G. and Akinyokun O. C., (2011), Design and Development of Multimedia Network System for e-Learning in Nigeria Universities.International Journal of Multimedia and Ubiquitous Engineering, Vol. 6, No. 3, pages 49-60.

[4]. Akinyokun O.C., Ubaru M.O. and Ezekwesili E., (2008), Information and Communications Technology for Development in Education Sector of Nigerian Economy.Proceedings of the International Conference on ICT Applications; Sponsored and Organized by Carnegie Corporation of New York and ObafemiAwolowo University, Ile-Ife, Nigeria.

[5]. Perraton H. (1998), A Theory of Distance Education. International perspectives Routledge. Pacific Mountain Network (producer), Far view I-IV [Videotape series]. Available from PMN, 1550 park avenue, Denver, CO 80218-1661, pp 34-45

[6]. Jonassen D. H. (1992), Applications and Limitations of hypertext technology for distance earning. Paper presented at the distance learning workshop, Armstrong Laboratory, San Antonio, TX.

[7]. Keegan D. (1990), Foundations of Distance Education, $2^{\text {nd }}$ New York: Routledge

[8]. Garrison D. R. and Shale D. (1997), Mapping the Boundaries of Distance Education: Problems in Defining the Field. The American Journal of Distance Education, Volume 1, Number 1, pages 7-13

[9]. Barron A. and Orwig G. (1993), New Technologies for Education.CO: Libraries Unlimited.

[10]. Holmberg B. (2005), A discipline of Distance Education. Journal of Distance Education, Lund Sweden Lector Publishing.

[11]. HarshitYadav, Kapil Sharma, RitikaDavial and SandeepRasgotra (2012), Virtual Classroom for National Institute of Technology, Hamirpur. A major Project Synopsissubmitted to Department of Computer Science and Engineering National Institute of Technology, Hamirpur, cse.nith.ac.in/Document/btech_mjrprojects/report/6.pdf, Accessed 11/06/2013.

[12]. Mahesh Maurya, ChitvanOza, AlokShah,Sadiya Patel (2011), Virtual Classroom System, www.nmims.edu/1809177872/virtual\% 20Classroom\%system_NCTET2011.pdf, Accessed 10/06/2013

[13]. Lertkulvanich Suriyong, Nithi Buranajant and SuwannaSombunsukho (2010), The Development of Virtual Classrooms by Using Virtual Image Creation Technique, International Journal of Human and Social Sciences Volume 5, Number 8

[14]. Obasa A.I (2010), Development of an Integrated Virtual Classroom System. MTech Thesis, Federal University of Technology, Akure.

[15]. ]Wichian Premchaiswadi, AnuchaTungkasthan, NipatJongsawat (2011), Enhancing Learning Systems by using Virtual Interactive ClassroomsandWeb-basedCollaborative www.ieec.uned.es/Investigacion/Educon2010/SearchTool/EDUCON2010/papers/2010S09A03.pdf, accessed 11/06/2013

[16]. Ibam Emmanuel Onwuka (2012), Web-Based Virtual Classroom System Model for Tertiary Institutions, Proceedings of the 2012 Summit of the African Educational Research Network, 18-20th May, 1 North Carolina State University \&DoubleTree Hilton, Raleigh Brownstone University Hotel

[17]. Chih-Yung Chang and Jang-Ping Sheu (2002), Design and Implementation of Ad Hoc Classroom and eSchoolbag Systems for Ubiquitous Learning. Proceedings of the IEEE International Workshop on Wireless and Mobile Technologies in Education (WMTE'02) 0-7695-1706-4/02 \$17.00, IEEE

[18]. Hsu Sam, Oge, Marques, M. Khalid Hamza, and BassemAlhalabi (2011), How to Design a Virtual Classroom: 10 Easy Steps to Follow, www.pps.teithe.gr/491995645/HowToDesignAVirtualClassroom.pdf, Accessed 10/06/2013

[19]. Zongkai Yang, Qingtang Liu (2007), Research and Development of Web-Based Virtual Online Classroom.ScienDirect, Computers \& Education Volume 48, 171-184

[20]. Elluminate (2007), Elluminate Live! Sessions.Elluminate Inc., Canada

[21]. Moodle (2005), Moodle for Teachers, Trainers and Administrators. Retrieved from: www.Moodle.org 